\title{
Comparison of radiation dose and its correlates between coronary computed tomography angiography and invasive coronary angiography in Northeastern Thailand
}

\author{
Phatraporn Aupongkaroon ${ }^{1}$, Pattarapong Makarawate ${ }^{2}$ and Narumol Chaosuwannakit ${ }^{{ }^{*}}$ (D)
}

\begin{abstract}
Background: The number of coronary computed tomography angiography (CCTA) exams is steadily growing. A novel computed tomography (CT) system has been developed to increase image quality while lowering patient radiation. The radiation dose attributed to CCTA has received considerable attention, whereas the dose associated with invasive catheter angiography (ICA) has received less. This study aims to investigate the radiation exposure of CCTA in patients and compare it to ICA.
\end{abstract}

Results: The mean effective dose of CCTA was $2.88 \pm 0.85 \mathrm{mSv}$ which was significantly lower than the mean effective dose of ICA (5.61 $\pm 0.55 \mathrm{mSv}$ ), $p<0.0001$. The effective dose of CCTA correlated with the weight, height, and BMI, while the effective dose of ICA was associated with patient weight and BMI. The radiation exposure from CCTA has been considerably reduced over the last ten years by almost 2.5 folds. The mean radiation dose from the newer generation CT used in 2019 was significantly lower than that of the single-source CT in $2010(2.88 \pm 0.85 \mathrm{mSv}$ vs. $7.15 \pm 3.4 \mathrm{mSv}$, $p<0.001$ ).

Conclusions: CCTA allows evaluation of CAD with a significantly less effective radiation dose to patients than diagnostic ICA. There was a significant decrease in radiation dose from CCTA over time. Regular measurement of patient doses is an essential step to optimize exposure. It makes operators aware of their performance and allows comparisons with generally accepted practices.

Keywords: Coronary CTA, CTA, Radiation dose, Coronary angiography

\section{Background}

The advancement of cardiac imaging modalities has resulted in significant improvements in the detection and treatment of cardiac disease in recent years. The coronary arteries have been visualized directly by coronary computed tomography angiography (CCTA) and invasive coronary angiography (ICA), which are the commonly used diagnostic modalities that involve ionizing radiation

\footnotetext{
*Correspondence: narumol_chao@yahoo.com

${ }^{1}$ Radiology Department, Faculty of Medicine, Khon Kaen University, Khon Kaen 40000, Thailand

Full list of author information is available at the end of the article
}

for assessing patients with possible coronary artery disease (CAD) (Fig. 1). The latter is considered the gold standard for the diagnosis of CAD $[1,2]$.

For imaging modalities that involve high doses or sensitive tissues in the primary radiation beam, the effective dose, absorbed dose, and organ doses are vital. Effective dose is a reasonable approximation of potential ionizing radiation damage and should be considered one parameter in determining the appropriateness of ionizing radiation examinations [2-4]. 


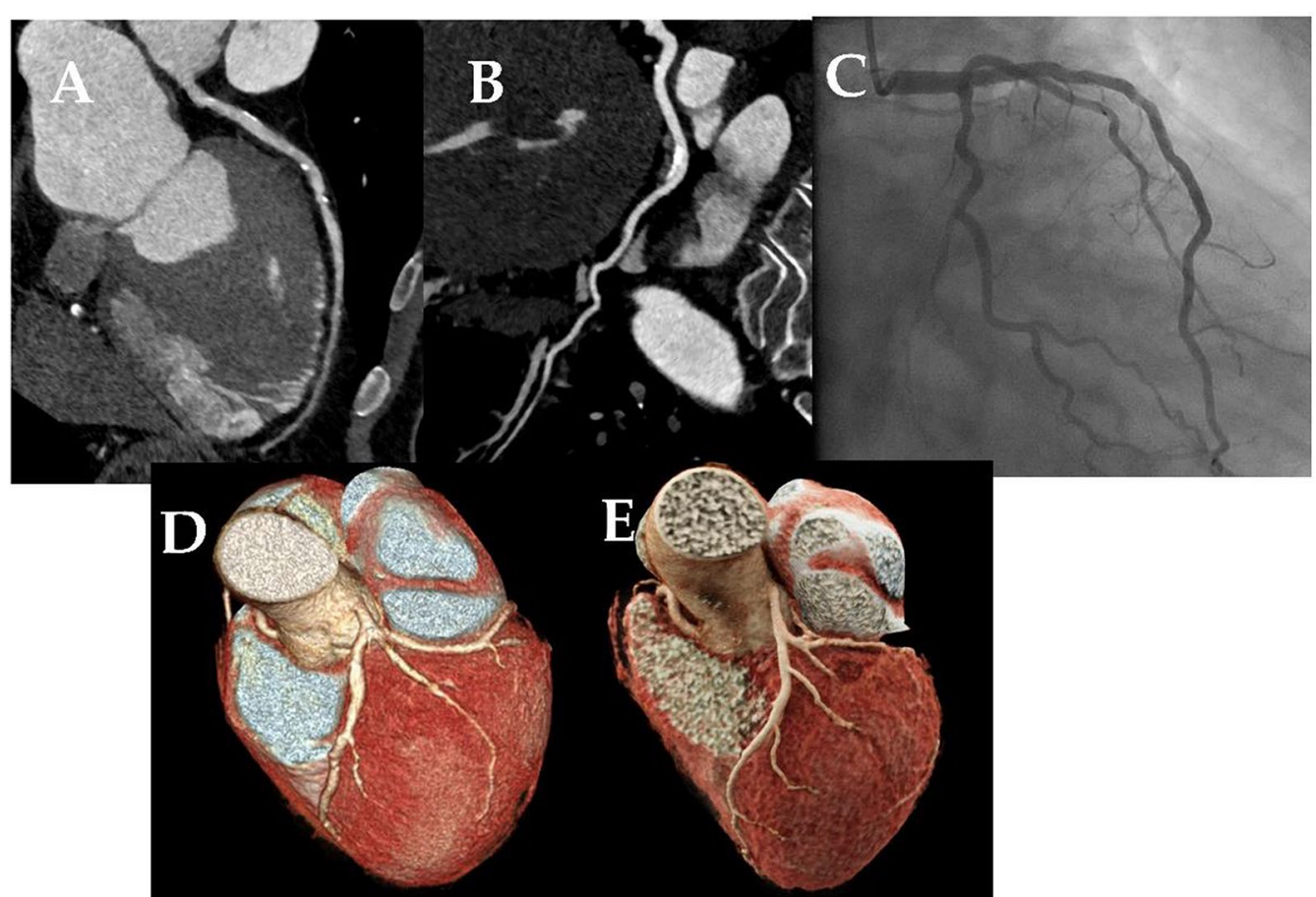

Fig. 1 Coronary CTA images from single- source CT scanner $(\mathbf{A}, \mathbf{D})$, and dual-source $C T$ scanner $(\mathbf{B}, \mathbf{E})$, and diagnostic invasive coronary angiography image $(\mathbf{C})$

To determine the effective dose, we evaluated the computed tomography dose index $\left(\mathrm{CTDI}_{\mathrm{vol}}\right)$ and dose-length product (DLP) from CCTA [2-4].

To estimate the radiation dose for diagnostic ICA, we assessed the air kerma-area product $\left(P_{\mathrm{KA}}\right)$ and expressed it in Gy.cm ${ }^{2}$, which is the recommended method to estimate the radiation dose for the patient in interventional cardiology [5].

This study aims to evaluate and compare the effective dose of CCTA and ICA and assess the correlation factors associated with high radiation dose and their advancement over time.

\section{Methods}

\section{Patient population}

We retrospectively reviewed patients' radiation doses who underwent CCTA and diagnostic ICA at Khon Kaen University Hospital, the leading teaching hospital and advanced tertiary care institution in northeastern Thailand, between January 2019 to December 2019. Patient identification was made by reviewing our institution's picture archiving and communication system (PACS) records data. The local Ethics Committee of Khon Kaen University, Thailand, reviewed and proved this study. The study was conducted according to the Declaration of Helsinki principles. All methods were performed following the relevant guidelines and regulations. The local Ethics Committee of Khon Kaen University also approved our investigation with a waiver of informed consent due to retrospective study design, and patient confidentiality was protected. Post-operative, post-revascularization, congenital heart disease patients, or the patients who underwent CCTA with increased field-of-view other than the standard scan range, and the missing radiation dose data in the picture archiving and communication system (PACS) were excluded from the present study.

\section{Coronary computed tomography angiography (CCTA)}

Coronary CTA exams were done (Definition FLASH dual-source CT, Siemens Healthcare, Forchheim, Germany). The system consists of two X-ray tubes and two detectors positioned on a single gantry with a 90-degree angular offset. Two X-ray sources, double sampling by fast longitudinal modification of the focused point (Z-flying focal spot), rotation period $330 \mathrm{~ms}$, automated tube voltage modulation were the CCTA scan standard protocol [6]. Patients were scanned while lying down. For the single-source CCTA exam, a 128-slice MDCT (Brilliance 128, Philips Healthcare, Netherland) used the following parameters: $128 \times 0.6$ collimation, 0.3 s rotation time, the pitch of $0.32,120 \mathrm{kV}$ tube voltage with ECG-triggering. 


\section{Diagnostic invasive coronary angiography (ICA)}

During the study period, 119 patients who underwent diagnostic ICA were recruited. The Philips Allura Xper biplane FD 10/10 (Eindhoven, Netherlands) was the two biplane angiocardiographic devices (double C-arc) in the catheterization lab at Khon Kaen University. The frame rate in normal fluoroscopy mode and digital cine acquisition was 15 frames per second. The system employs a sophisticated automatic dose control system for fluoroscopy automated spectrum beam filter selection.

\section{Definition and dosimetry}

For each CCTA, the CTDI ${ }_{\mathrm{vol}}$ and dose length product (DLP) were recorded. The patient dose data, CT dose index volume $\left(\mathrm{CTDI}_{\mathrm{vol}}\right)$, and dose length product (DLP) values, were extracted from the picture archiving and communication system (PACS). The Dose Length Product (DLP, units: $\mathrm{mGy} . \mathrm{cm}$ ) indicates the mean absorbed dose to the patient of each sequence in a CT exam and is calculated by multiplying CTDI $\mathrm{vol}_{\text {by }}$ the scan length. It measures the total CT examination's mean effective dose to the patient $[7,8]$. The CT monitor's real-time CTDI $_{\text {vol }}$ and DLP displays were collected in the PACS and retrospectively analyzed. For ICA, the air kermaarea product $\left(P_{\mathrm{KA}}\right)$ meters quantify radiation dose in the unit and cumulative air kerma. The $P_{\mathrm{KA}}$ meters integrate exposure throughout the entire image field using an air ionization chamber installed in the X-ray assembly. The biplane's total dose was determined in units of $P_{\mathrm{KA}}\left(\mathrm{Gy} . \mathrm{cm}^{2}\right)$ [5]. The effective radiation dose was determined to $\mathrm{mSv}$ and compared imaging modalities based on current publications and manufacturer information. A factor of $0.014 \mathrm{mSv} / \mathrm{mGy} . \mathrm{cm}$ was used to convert the CCTA dose-length product $[9,10]$, whereas $0.018 \mathrm{mSv} / \mathrm{Gy} \cdot \mathrm{cm}^{2}$ was used to convert the ICA air kerma-area product $[5,11]$. The effective radiation dose was compared each year to assess the variation of radiation doses over time. In an attempt to discover predictors of dose change over time, all prospectively

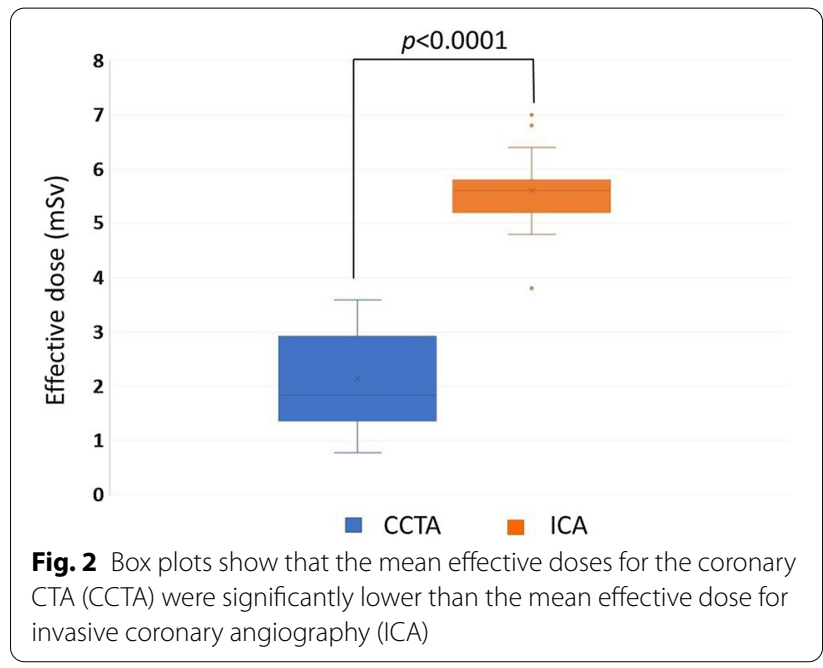

collected variables in the respective databases were analyzed.

\section{Statistical analysis}

SPSS Statistics 17.0 for Windows was used to accomplish the statistical analysis. Unless otherwise stated, continuous variables are reported as mean and standard deviation, and categorical variables are presented as number (n) or frequency (percent). The nonparametric MannWhitney or Kruskal-Wallis tests were used to evaluate continuous variables. Spearman correlation coefficient was used to assess the correlation between the effective dose and patient characteristic factors. The chi-square test was used to compare frequency distributions. Twosided $p$ values of less than 0.05 were considered statistically significant.

\section{Results}

During the retrospective study period, 406 CCTA studies and 119 diagnostic ICA were recruited. The mean effective dose of CCTA performed between January to December 2019 was $2.88 \pm 0.85 \mathrm{mSv}$, significantly lower than the mean effective dose of diagnostic ICA ( $5.61 \pm 0.55 \mathrm{mSv}$ ), $p<0.0001$ (Table 1). For ICA, the mean

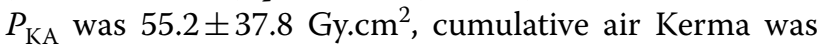

Table 1 Radiation dose from coronary CTA (CCTA) and invasive diagnostic coronary angiography (ICA)

\begin{tabular}{llll}
\hline Parameters & CTA $(\boldsymbol{n = 4 0 6 )}$ & ICA $(\boldsymbol{n}=\mathbf{1 1 9})$ & $\boldsymbol{P}$ value (95\% Cl) \\
\hline Effective dose $(\mathrm{mSv})$, mean \pm SD & $2.88 \pm 0.85$ & $5.61 \pm 0.55$ & $<0.0001^{*}(2.43-3.02)$ \\
Gender, (male, \%) & $24(30.7)$ & $14(35.9)$ & $0.70(-17.09$ to 32.28$)$ \\
Age $($ years), mean \pm SD & $65.5 \pm 9.6$ & $65.8 \pm 9.4$ & $0.87(-3.40$ to 4.0$)$ \\
BMI $\left(\mathrm{kg} / \mathrm{m}^{2}\right)$, mean \pm SD & $23.4 \pm 3.6$ & $23.1 \pm 3.4$ & $0.66(-1.67$ to 1.07$)$ \\
\hline
\end{tabular}

*Statistically significant at the $p$ value $<0.05$ 
Table 2 Correlation between patient characteristics and effective dose of coronary CTA

\begin{tabular}{lcl}
\hline & Correlation coefficient $(\boldsymbol{r})$ & $\boldsymbol{P}$ value \\
\hline Age & -0.25 & 0.273 \\
Weight & 0.42 & $0.000129^{*}$ \\
Height & 0.27 & $0.017^{*}$ \\
BMl & 0.387 & $0.000465^{*}$ \\
\hline
\end{tabular}

* Pearson correlation is significant at the $p$ value $<0.05$ [2-tailed]

Table 3 Correlation between patient characteristics and effective dose of ICA

\begin{tabular}{lcl}
\hline & Correlation coefficient $(\boldsymbol{r})$ & $\boldsymbol{P}$ value \\
\hline Age & -0.04 & 0.808 \\
Weight & 0.29 & $0.04^{*}$ \\
Height & 0.18 & 0.27 \\
BMl & 0.24 & $0.0116^{*}$
\end{tabular}

*Pearson correlation is significant at the $p$ value $<0.05$ [2-tailed] techniques and heart rates, and the mean effective dose is $7.15 \pm 3.4 \mathrm{mSv}$ [12]. Compared to the present study, we documented a significant decrease in radiation dose by CCTA over time and identified the correlation of a higher radiation dose. The radiation exposure from CCTA has been considerably reduced over the last ten years by almost 2.5 folds. The mean CTDI ${ }_{\text {vol }}$ and DLP from the newer generation CT used in 2019-2020 was significantly lower than that of the single-source CT in $2010(9.8 \pm 2.7 \mathrm{mGy}$ vs. $36.7 \pm 7.8 \mathrm{mGy}, p<0.0001$ and $188 \pm 46 \mathrm{mGy.cm}$ vs. $584 \pm 98 \mathrm{mGy.cm}, p<0.0001)$ (Table 4; Fig. 3).

\section{Discussion}

Our study discovered a wide range of effective radiation doses associated with routine cardiovascular diagnostic procedures. The radiation dose for diagnostic ICA was significantly higher, whereas the dose for CCTA was considerably lower. We also uncovered CCTA-related

Table 4 Comparison of radiation dose and patient characteristics between dual-source CTA (DSCTA) in 2019 and single-source CTA (SSCTA) in 2010

\begin{tabular}{llll}
\hline Parameters & DSCTA $(\boldsymbol{n = 4 0 6 )}$ & SSCTA $(\boldsymbol{n}=\mathbf{2 3 3})$ & $\boldsymbol{P}$ value (95\% CI) \\
\hline Effective dose $(\mathrm{mSv})$, mean \pm SD & $2.88 \pm 0.85$ & $7.15 \pm 3.4$ & $<0.0001^{*}(3.64-4.89)$ \\
Gender, (male,\%) & $24(30.7)$ & $9(47.4)$ & $0.14(-4.57$ to 38.55$)$ \\
Age (years), mean \pm SD & $65.5 \pm 9.6$ & $68.3 \pm 10.2$ & $0.06(-9.79$ to -0.60$)$ \\
BMI (kg/m²), mean \pm SD & $23.4 \pm 3.6$ & $22.9 \pm 5.5$ & $0.58(-2.3$ to 1.32$)$ \\
\hline
\end{tabular}

*Statistically significant at the $p$ value $<0.05$

$782 \pm 394.8 \mathrm{mGy}$, and the mean fluoroscopic time was $8.8 \pm 10.9 \mathrm{~min}$. The effective dose comparing between procedures by box plots is shown in Fig. 2 .

When we investigated the correlation between the effective dose and patient characteristics for CCTA, the results showed a strong linear relationship between the effective dose and body weight $(r=0.42, p<0.001)$, BMI $(r=0.387, p<0.001)$, and a good linear relationship between the effective dose and height $(r=0.27$, $p=0.017$ ), respectively (Table 2 ).

For ICA, we found a poor linear relationship with no significant between the effective dose and patient age and height. The results also demonstrated an excellent linear relationship between the effective dose and BMI $(r=0.24$, $p=0.0116)$ and a fair relationship between the effective dose and weight $(r=0.29, p=0.04)$ as demonstrated in Table 3 for the Spearman correlation coefficient.

We have previously reported on the effective radiation dose associated with CCTA performed in 2010 in a single-center experience demonstrated the effective radiation dose of coronary CTA ranged from 2.8 to $11.5 \mathrm{mSv}$ depended on different dose-saving

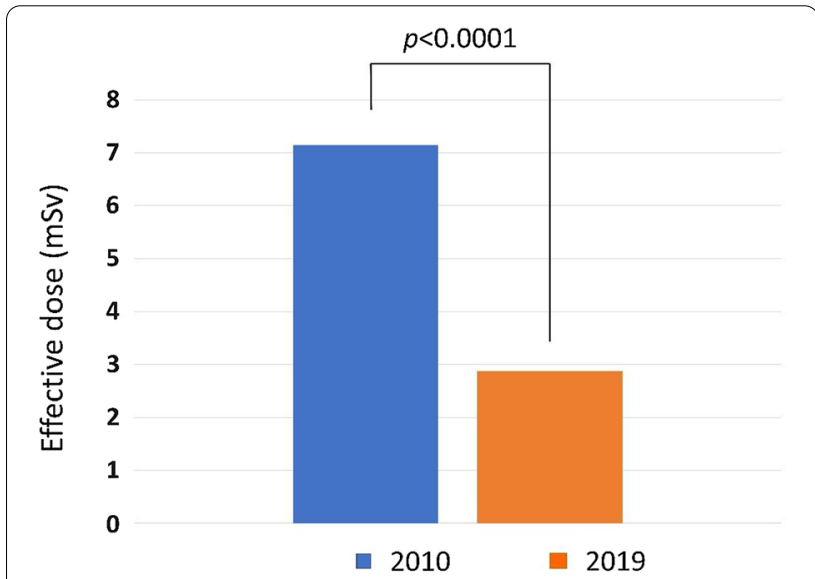

Fig. 3 Box plots show that the mean effective doses for the coronary CTA (CCTA) performed in 2019 were significantly lower than the mean effective dose for CCTA in 2010

trends in the mean effective radiation dose and a relationship between radiation dose and certain clinical 
factors. There is a gross lack of information on radiation doses to patients in most Asian countries and the absence of reports from Thailand on this issue.

To our knowledge, the present study is the first investigation of comparing the patient radiation dose during various diagnostic cardiac procedures in Thailand. We hope it may stimulate interest in the area to benefit both patients and staff.

In Northeastern Thailand, this is the first report of radiation dose from diagnosis ICA utilizing the two biplane angiocardiographic devices (double $\mathrm{C}$-arc). The results of this study will promote radiation dose optimization and benefits in both patients and staff.

We have previously reported on the effective radiation dose associated with CCTA performed in 2010 in a single-center experience demonstrated the effective whole-body dose of CCTA ranged from 2.8 to $11.5 \mathrm{mSv}$ depended on different dose-saving techniques and heart rates. The mean effective dose is $7.15 \pm 3.4 \mathrm{mSv}$. For patients in the prospective ECG-triggering (PT) group, the mean DLP was $184 \pm 66$ mGy.cm, resulting in an effective radiation dose per examination of $3.1 \pm 1.1 \mathrm{mSv}$. In the retrospective (RG) group, the mean DLP was $501 \pm 198 \mathrm{mGy} \mathrm{cm}$, resulting in an effective radiation dose per examination of $8.5 \pm 3.4 \mathrm{mSv}$. Compared to the present study, we documented a significant decrease in radiation dose by CCTA over time and identified the correlation of a higher radiation dose. The radiation exposure from CCTA has been considerably reduced over the last ten years by almost 2.5 folds. The mean radiation dose from the newer generation CT used in 2019 was significantly lower than that of the single-source $\mathrm{CT}$ in 2010, $p<0.0001$. In 2010, the median effective dose for coronary CTA was $7.15 \pm 3.4 \mathrm{mSv}$. Still, by 2019 , it has dropped to $2.88 \pm 0.85 \mathrm{mSv}$, resulting in a $59.7 \%$ reduction in radiation exposure or over 2.5 times reduction in the effective dose $(p<0.0001)$.

Notably, the number of non-diagnostic coronary CTAs did not rise over the study period, remaining below $3 \%$ in 2010 and 2019. As the number of detector slices increases and with faster gantry rotation speeds, the temporal and spatial resolutions improve cardiac imaging, consistent with the prior study by Liang et al. [13] that coronary CTA performed on dual-source CT results in better image quality lower radiation dose than single-slice CT.

On this concept, ionizing radiation procedures should be conducted with the "as low as reasonably achievable" philosophy in consideration, and clinicians ordering and conducting cardiac imaging diagnostic tests should be knowledgeable with the associated radiation doses and strategies for reducing them. The mean effective radiation dose we discovered for each exam corresponds with previous studies with the same dual-source CT scanner
$[14,15]$. Kosmala et al. [14] studied the radiation dose of CCTA with a third-generation dual-source scanner in a real-world patient population and demonstrated the median effective dose was $1.32 \mathrm{mSv}$ for prospective sequence and $4.77 \mathrm{mSv}$ for retrospective sequence.

Furthermore, we evaluated certain variables that influence the effective radiation dose delivered by these exams. Obese individuals received considerably greater mean effective radiation doses in all the exams investigated. This was especially true of CCTA and ICA. For CCTA, the effective radiation dose was correlated with higher weight, BMI, and height, demonstrated by another study [16]. For ICA, the effective radiation dose correlated with higher BMI and weight, in line with published data [17].

There are some limitations in the present study. Firstly, the current study is retrospective, single-center experience; hence, further prospective study with larger recruited patients should be considered.

Secondly, because of several technical advancements, the CCTA has significantly reduced radiation dose, which can currently be reached at the submillisievert level.

It's important to remember that ICA has been following these developments, which were not included in this study. Because of decreased frame rates and magnification, appropriate collimation, and software solutions that interpolate virtual images between frames, ICA radiation exposure has been significantly reduced in the contemporary catheterization laboratory during the last decade.

Finally, the ICA registry's higher radiation dose was also correlated to a higher number of positive and complexity of coronary artery disease. However, we did not analyze this. We can assume that the complexity of conditions needed more cine angiograms of the coronary arteries, with a consequent increase in the radiation dose used $[18,19]$.

\section{Conclusions}

CCTA provides a comprehensive CAD assessment, yet it exposed patients to a substantially lower effective radiation dose than diagnostic ICA.

The radiation exposure from CCTA decreased significantly over ten years period.

Measuring patient doses on a regular schedule is critical for optimizing exposure.

It promotes operator awareness of their performance and allows for comparisons with generally accepted practices.

\section{Abbreviations}

CCTA: Coronary computed tomography angiography; ICA: Invasive coronary angiography; CAD: Coronary artery disease; CTDI vol: Computed tomography 
dose index; DLP: Dose-length product; $\mathrm{P}_{\text {KA }}$ : Air kerma-area product; PACS: Picture archiving and communication system.

\section{Acknowledgements}

We wish to thank the patients and families for their cooperation. We also would like to thank the cardiology staff at the department of medicine for their patient care services. The authors thank the Department of Radiology, Internal medicine, and Faculty of Medicine for their support.

\section{Authors' contributions}

PA involved data collection, reviewing medical records, and radiation doses. PM involved in drafting the manuscript. NC involved study design, data collection, review radiation doses and medical records, statistical analysis, data interpretation, drafted the manuscript. All authors read and approved the final manuscript.

\section{Funding}

None.

\section{Availability of data and materials None.}

\section{Declarations}

\section{Ethics approval and consent to participate}

The local Ethics Committee of Khon Kaen University, Thailand, reviewed and proved this study and was registered under reference number HE641421. All methods were performed following the relevant guidelines and regulations. The local Ethics Committee of Khon Kaen University approved our investigation with a waiver of informed consent due to the retrospective study design.

\section{Consent for publication}

Not applicable.

\section{Competing interests}

The authors declare that they have no known competing financial interests or personal relationships that could have influenced the work reported in this paper.

\section{Author details}

${ }^{1}$ Radiology Department, Faculty of Medicine, Khon Kaen University, Khon Kaen 40000, Thailand. ${ }^{2}$ Cardiology Unit, Internal Medicine Department, Faculty of Medicine, Khon Kaen University, Khon Kaen, Thailand.

Received: 14 December 2021 Accepted: 14 January 2022

Published online: 25 January 2022

\section{References}

1. Wijns W, Kolh P, Danchin N, Mario CD, Falk V, Folliguet T et al (2010) Task force on myocardial revascularization of the European Society of Cardiology (ESC), the European Association for Cardio-Thoracic Surgery (EACTS), European Association for Percutaneous Cardiovascular Intervention (EAPCI), Guidelines on myocardial revascularization. Eur Heart J $31: 2501-2555$

2. Gerber TC, Carr JJ, Arai AE, Dixon RL, Ferrari VA, Gomes AS et al (2009) lonizing radiation in cardiac imaging: a science advisory from the American Heart Association Committee on Cardiac Imaging of the Council on Clinical Cardiology and Committee on Cardiovascular Imaging and Intervention of the Council on Cardiovascular Radiology and Intervention. Circulation 119:1056-1065

3. Einstein AJ, Moser KW, Thompson RC (2007) Radiation dose to patients from cardiac diagnostic imaging. Circulation 116:1290-1305

4. Hausleiter J, Meyer T, Hermann F, Hadamitzky M, Krebs M, Gerber TC et al (2009) Estimated radiation dose associated with cardiac CT angiography. JAMA 301:500-507

5. Fazel R, Krumholz HM, Wang Y, Ross JS, Chen J, Ting HH et al (2009) Exposure to low-dose ionizing radiation from medical imaging procedures. $\mathrm{N}$ Engl J Med 361:849-857
6. Chaosuwannakit N, Makarawate P (2021) Diagnosis and prognostic significance of anomalous origin of coronary artery from the opposite sinus of Valsalva assess by dual-source coronary computed tomography angiography. IJC Heart Vascul 32(2):100723

7. Griffey RT, Sodickson A (2009) Cumulative radiation exposure and cancer risk estimates in emergency department patients undergoing repeat or multiple CT. AJR Am J Roentgenol 192:887-892

8. Sodickson A, Baeyens PF, Andriole KP, Prevedello LM, Nawfel RD, Hanson R et al (2009) Recurrent CT, cumulative radiation exposure, and associated radiation-induced cancer risks from CT of adults. Radiology 251:175-184. https://doi.org/10.1148/radiol.2511081296

9. Hausleiter J, Meyer T, Hermann F et al (2009) Estimated radiation dose associated with cardiac CT angiography. JAMA 301:500-507

10. Chaosuwannakit N, Makarawate P (2018) Reduction of radiation dose for coronary computed tomography angiography using prospective electrocardiography-triggered high-pitch acquisition in clinical routine. Pol J Radiol 83:e260-e267

11. Neill J, Douglas H, Richardson G, Chew EW, Walsh S, Hanratty C et al (2010) Comparison of radiation dose and the effect of operator experience in femoral and radial arterial access for coronary procedures. Am J Cardiol 106:936-940

12. Chaosuwannakit N, Makarawate $P$ (2013) Comparison of patient radiation dose for 128-row multidetector coronary computed tomography angiography by prospective versus retrospective ECG-triggering techniques. Srinagarind Med J 28(3):287-293

13. Liang CR, Ong CC, Chai P, Teo LS (2021) Comparison of radiation dose, contrast enhancement and image quality of prospective ECG-Gated CT coronary angiography: single versus dual source CT. Radiography 27(3):831-839

14. Kosmala A, Petritsch B, Weng AM, Bley TA, Gassenmaier T (2019) Radiation dose of coronary CT angiography with a third-generation dual-source CT in a "real-world" patient population. Eur Radiol 29:4341-4348

15. Li RF, Hou CL, Zhou H, Dai YS, Jin LQ, Xi Q et al (2020) Comparison on radiation effective dose and image quality of right coronary artery on prospective ECG-gated method between 320 row CT and 2nd generation (128-Slice) dual-source CT. J Appl Clin Med Phys 21(8):256-262

16. Sabarudin A, Siong TW, Chin AW, Hoon NK, Karim MK (2019) A comparison study of radiation effective dose in ECG-gated coronary CT angiography and calcium scoring examinations performed with a dual-source CT scanner. Sci Rep. https://doi.org/10.1038/s41598-019-40758-5

17. Crowhurst JA, Whitby M, Savage M, Murdoch D, Robinson B, Shaw E et al (2019) Factors contributing to radiation dose for patients and operators during diagnostic cardiac angiography. J Med Radiat Sci 66(1):20-29

18. Crowhurst JA, Whitby M, Thiele D, Halligan T, Westerink A, Crown S et al (2014) Radiation dose in coronary angiography and intervention: initial results from the establishment of a multi-center diagnostic reference level in Queensland public hospitals. J Med Radiat Sci 61(3):135-141

19. Tsapaki V, Ahmed NA, AISuwaidi JS, Beganovic A, Benider A, BenOmrane $L$ et al (2009) Radiation exposure to patients during interventional procedures in 20 countries: initial IAEA project results. AJR Am J Roentgenol 193:559-569

\section{Publisher's Note}

Springer Nature remains neutral with regard to jurisdictional claims in published maps and institutional affiliations. 